\title{
Human Audiometric Thresholds do not Predict Specific Cellular Damage in the Inner Ear
}

Lukas D. Landegger ${ }^{1,2,3}$, Demetri Psaltis ${ }^{4}$, Konstantina M. Stankovic ${ }^{1,2,5^{*}}$

${ }^{1}$ Eaton Peabody Laboratories, Department of Otolaryngology, Massachusetts Eye and Ear Infirmary, 243 Charles St, Boston, MA 02141, United States of America

${ }^{2}$ Department of Otolaryngology, Harvard Medical School, 25 Shattuck St, Boston, MA 02115, United States of America

${ }^{3}$ Department of Otolaryngology, Vienna General Hospital, Medical University of Vienna, Waehringer Guertel 18-20, 1090 Vienna, Austria

${ }^{4}$ Optics Laboratory, School of Engineering, Swiss Federal Institute of Technology Lausanne (EPFL), BM 4102 (Bâtiment BM), Station 17, 1015 Lausanne, Switzerland ${ }^{5}$ Harvard Program in Speech and Hearing Bioscience and Technology, 260 Longwood Avenue, Boston, MA 02115, United States of America

* Corresponding author

E-mail: konstantina_stankovic@meei.harvard.edu (KMS)

E-mail addresses of the other authors:

lukas_landegger@meei.harvard.edu

demetri.psaltis@epfl.ch

Abbreviations: ERB, Equivalent rectangular bandwidth; HL, Hearing level; PTA, Pure tone average; SNHL, Sensorineural hearing loss 


\section{Abstract}

Introduction: As otology enters the field of gene therapy and human studies commence, the question arises whether audiograms - the current gold standard for the evaluation of hearing function - can consistently predict cellular damage within the human inner ear and thus should be used to define inclusion criteria for trials. Current assumptions rely on the analysis of small groups of human temporal bones post mortem or from psychophysical identification of cochlear "dead regions" in vivo, but a comprehensive study assessing the correlation between audiometric thresholds and cellular damage within the cochlea is lacking.

Methods: A total of 131 human temporal bones from 85 adult individuals (ages 1992 years, median 69 years) with sensorineural hearing loss due to various etiologies were analyzed. Cytocochleograms - which quantify loss of hair cells, neurons, and strial atrophy along the length of the cochlea - were compared with subjects' latest available audiometric tests prior to death (time range 5 hours to 22 years, median 24 months). The Greenwood function and the equivalent rectangular bandwidth were used to infer, from cytocochleograms, cochlear locations corresponding to frequencies tested in clinical audiograms. Correlation between audiometric thresholds at clinically tested frequencies and cell type-specific damage in those frequency regions was examined by calculating Spearman's correlation coefficients. Results: Similar audiometric profiles reflected widely different cellular damage in the cochlea. In our diverse group of patients, audiometric thresholds tended to be more influenced by hair cell loss than by neuronal loss or strial atrophy. Spearman's correlation coefficient across frequencies was at most 0.7 and often below 0.5 , with 1.0 indicating perfect correlation. 
Conclusions: Audiometric thresholds do not predict specific cellular damage in the human inner ear. Our study highlights the need for better non- or minimally-invasive tools, such as cochlear endoscopy, to establish cellular-level diagnosis and thereby guide therapy and monitor response to treatment.

Keywords: Cytocochleograms; audiometric thresholds; human temporal bones; hair cells; stria vascularis; spiral ganglion neurons

\section{Introduction}

Hearing loss is the most common sensory deficit in humans and a major global health challenge. Three hundred and sixty million people are currently affected by moderate to profound hearing loss, and the increasing use of personal listening devices, mass attendance at sporting events and concerts, and the aging of a populous generation place 1.1 billion more at risk (Daniel, 2007; Wallhagen et al., 1997). The underlying causes of hearing loss are diverse and include age, genetics, infection, trauma and exposure to noise or ototoxic drugs. While most of the conductive forms of hearing loss - which affect transmission at the level of the middle ear - can be adequately treated with surgery or medications, there is still no cure for sensorineural hearing loss (SNHL), which reflects damage to the delicate mechanosensory structures of the inner ear (Geleoc et al., 2014). Therapeutic approaches to SNHL have rapidly evolved over the last few decades and now include astonishingly successful electronic devices, like cochlear and auditory 
brainstem implants. However, these devices provide a different and limited perception of sound and speech compared to that provided by "natural hearing" (Carlson et al., 2012).

To address limitations of the current rehabilitative approaches for SNHL, new strategies are being developed and include the administration of genes, small molecules and stem cells directly to the inner ear, with clinical trials currently ongoing and results yet to be published (Chien et al., 2015). For such approaches to be successful, it is of utmost importance to identify the extent of cochlear damage at the cellular level so to develop precise and personalized therapies.

Inner hair cells are the main sensory cells in the inner ear, and they perform mechanotransduction - the conversion of a mechanical stimulus into an electrical response, which is then processed by spiral ganglion neurons and the central nervous system. Outer hair cells are motile and they amplify the traveling wave in the cochlea to provide exquisite sensitivity of the hearing organ. Stria vascularis is a vascular structure in the cochlear lateral wall and generates the endocochlear potential that drives transduction current through hair cells.

The vast majority of human studies on hearing restoration rely on the wellestablished analysis of standardized audiograms, which reflect hearing thresholds in quiet as a function of frequency. Some studies include additional indirect metrics like word recognition scores, defined as the percentage of words a patient can correctly repeat after listening to a standardized word list in quiet; otoacoustic emissions, which are generated by outer hair cells and serve as a measure of those cells' integrity; and auditory brainstem responses, which are surface potentials consisting of several waves, the first of which reflects the summed activity of the cochlear nerve (Causey et al., 1984; Gelfand, 1997; Thornton et al., 1978). However, although it is common in animal studies to verify the consequences of experimental procedures 
with histological data, very little is known about the predictive value of human audiometric thresholds to detect specific cellular damage in the inner ear.

Capitalizing on the precious resources of the US Temporal Bone Registry, which has one of the world's largest collections of human post-mortem temporal bones, we sought to determine to what extent human audiograms predict changes in cochlear histopathology.

\section{Materials and methods}

\subsection{Temporal bone preparation and study}

The archival collection of human temporal bones from the US Temporal Bone Registry at Massachusetts Eye and Ear Infirmary was inspected to identify the specimens that had been quantified using cytocochleograms, which are graphic representations of structural integrity of sensory hair cells, cochlear neurons and stria vascularis along the cochlear length (see Fig. A.1) (Schuknecht, 1968). A total of 131 temporal bones from 85 hearing-impaired adult patients (age range 19-92 years, median 69 years; see Fig. A.2A) were analyzed. The most common diagnosis was presbycusis, i.e. age-related hearing loss (22 ears), followed by a combination of presbycusis and acoustic trauma (11 ears), kanamycin ototoxicity (7 ears), sudden SNHL (6 ears), "isolated" SNHL (6 ears), otosclerosis (5 ears) and several rare diseases and syndromes (see Table A.1). Written informed consent had been obtained prior to death and the study was carried out according to The Code of Ethics of the World Medical Association (Declaration of Helsinki). The current study was approved by the institutional review board. As previously described by Schuknecht, bones were removed after death (post-mortem time range 2-63 hours, 
median 10 hours, no information for 4 subjects; see Fig. A.2B) and fixed in 10\% neutral buffered formalin or Heidenhain Susa solution, decalcified in ethylenediaminetetraacetic acid, embedded in celloidin, serially sectioned at a thickness of $20 \mu \mathrm{m}$ in the horizontal (axial) plane, and every tenth section was stained with hematoxylin and eosin (Schuknecht, 1968). The specimens on glass slides were examined using light microscopy. Cytocochleograms were created to quantify fractional loss of hair cells and cochlear neurons as well as strial atrophy along the cochlear spiral after standardizing cochlear length to 32 millimeters for all ears (Schuknecht, 1993). Up to 320 different data points per cochlea (one every 100 micrometers) were collected. The resulting cytocochleograms were sorted from most to least extensive damage for every cell type to generate waterfall plots (Figs 1-5). Earlier cytocochleograms combined inner and outer hair cells into "hair cells," while more recent cytocochleograms classify inner and outer hair cells separately.

\subsection{Hearing tests}

Standardized pure tone audiometric thresholds, identified at 250, 500, 1000, 2000, 4000 and $8000 \mathrm{~Hz}$, were determined as a part of the routine clinical examination by increasing sound level in $5 \mathrm{~dB}$ increments from $0 \mathrm{~dB}$ to a maximum of $100 \mathrm{~dB}$. The current study focused on the latest available audiograms recorded for each patient prior to death, which ranged from 5 hours to 22 years, with a median of 24 months (see Fig. A.2D). Three individuals did not undergo a standardized hearing test, but were diagnosed with profound deafness during clinical examination. For one person, the date of audiologic testing was not specified. Pure tone average (PTA) was noted, defined as the average $\mathrm{dB}$ hearing level of the two frequencies with the lowest thresholds in the frequency range from 500 to $2000 \mathrm{~Hz}$ as this provides the closest 
agreement with speech reception thresholds (Fletcher, 1953). PTA and damage at the corresponding position in the cytocochleogram were compared according to tonotopic arrangement along the length of the cochlea. Each audiogram was compressed into a linear heat map, with the color reflecting $\mathrm{dB}$ of hearing loss across increasing frequencies; an increasing gray scale was used for increasing $\mathrm{dB}$ of hearing loss from $0 \mathrm{~dB}$ (white) to $100 \mathrm{~dB}$ or no response (black) (see Fig. A.1B). Word recognition scores were available and analyzed for 70 temporal bones from 44 patients (see Fig. A.2C).

\subsection{Correlation of cellular damage and audiograms}

The frequency, $f$, corresponding to a specific location along the cytocochleograms, $x$, was calculated according to a modified Greenwood function: $f=165.4\left(10^{2.1 x}-0.88\right)$ (Greenwood; Greenwood). The cochlear region responding to a certain frequency was determined using the equivalent rectangular bandwidth (ERB), a measurement defined in psychoacoustic studies to represent an approximation of the bandwidths of the filters in human hearing (Glasberg et al., 1990; Moore et al., 1983). In humans, the ERB reflects a constant distance of $0.9 \mathrm{~mm}$ on the basilar membrane.

Consequently, we calculated the average damage for each location corresponding to a specific frequency, +/- $0.45 \mathrm{~mm}$ (Moore, 1986). For word recognition scores, we combined all frequency-specific results and weighted them based on the octave-band Speech Intelligibility Index described by the American National Standard (ANSI S3.51997) (e.g. damage in the area of $250 \mathrm{~Hz}$ was weighted by 0.0617 , damage in the area of $500 \mathrm{~Hz}$ was weighted by 0.1671 , etc.) (ANSI, 1997). Spearman's correlation coefficients between audiometric thresholds and degree of cell-specific damage in the respective areas were calculated. For word recognition scores, the number (\% correct) was transformed (original value subtracted from 100) before performing 
calculations to visualize a positive correlation $(100=$ worst, $0=$ best; $1=$ most and 0 = least damage) and simplify comparison with the frequency-specific results (100 dB $=$ worst, $0 \mathrm{~dB}=$ best $)$. The computations were performed with $\mathrm{R}(\mathrm{R}$ Core Team) and $p<0.05$ was considered significant after Benjamini-Hochberg correction for multiple comparisons. Graphs were generated using MATLAB (MathWorks, Natick, MA) and Microsoft Excel (Microsoft, Redmond, WA), as well as GNU-based counterparts R ( $R$ Core Team), GIMP (GIMP Development Team) and Inkscape (Inkscape Team).

\section{Results}

\subsection{Cell type-specific damage}

Cytocochleograms depicting combined loss of inner and outer hair cells (Fig 1), loss of inner hair cells alone (Fig 2), loss of outer hair cells alone (Fig 3), strial atrophy (Fig 4), or loss of spiral ganglion neurons (Fig 5) demonstrated a wide range of damage across patients and frequencies.

Fig 1. Combined hair cell analysis. Overview of an analysis of 70 human temporal bones sorted according to the degree of histological damage at the level of inner and outer hair cells $(\mathbf{A})$ and the corresponding most recent audiogram prior to death (B) listed in the same order. Color in the heat map $(B)$ reflects $\mathrm{dB}$ of hearing level $(\mathrm{HL})$, with white indicating normal hearing $(0 \mathrm{~dB} \mathrm{HL})$ and black indicating profound deafness (100 dB HL); stripes depict responses that were not measured.

Fig 2. Inner hair cell analysis. Overview of an analysis of 56 human temporal bones sorted according to the degree of histological damage at the level of inner hair cells 
(A) and the corresponding most recent audiogram prior to death (B) listed in the same order. Color conventions as in Fig 1.

Fig 3. Outer hair cell analysis. Overview of an analysis of 57 human temporal bones sorted according to the degree of histological damage at the level of outer hair cells (A) and the corresponding most recent audiogram prior to death (B) listed in the same order. Color conventions as in Fig 1.

Fig 4. Stria vascularis analysis. Overview of an analysis of 100 human temporal bones sorted according to the degree of histological damage of the stria vascularis (A) and the corresponding most recent audiogram prior to death (B) listed in the same order. Color conventions as in Fig 1.

Fig 5. Spiral ganglion neuron analysis. Overview of an analysis of 127 human temporal bones sorted according to the degree of spiral ganglion neuron loss $(\mathbf{A})$ and the corresponding most recent audiogram prior to death (B) listed in the same order. Color conventions as in Fig 1.

Combined damage of inner and outer hair cells was quantified in 70 ears (Fig 1A) and ranged from no hair cell loss to hair cell annihilation. The latter is exemplified by patient 26 , who had received 1.5 grams of kanamycin for a period of eight days (total dosage $12 \mathrm{~g}$ ) to treat severe pyelonephritis accompanying a metastatic adenocarcinoma of the cervix in the 1960s. In 11 out of 17 patients, the degree of loss in one ear was within $10 \%$ of loss in the other ear.

If cellular damage and audiometric thresholds correlated perfectly, we would expect a clear transition from black (most damaged specimen at the top of the audiometric heat map) to white (least damaged specimens at the bottom of the audiometric heat 
map) (see Fig. A.1C). However, there is no obvious correlation between the degree of cellular damage and audiometric thresholds when corresponding audiograms are depicted in the same order as cytocochleograms (Fig 1B).

When analyzing inner hair cells specifically, quantified in 56 ears (Fig 2A), the spectrum of damage again ranged from no loss to complete loss. The latter category included a patient (number 46) who had suffered a left permanent sudden sensorineural hearing loss in the setting of ipsilateral chronic otitis media with cholesteatoma; in the contralateral ear, a reversible sudden sensorineural hearing loss resulted in only $1 \%$ loss of inner hair cells. In 9 out of 15 patients, the degree of inner hair cell loss in one ear was within $10 \%$ of loss in the other ear. An obvious correlation between histological (Fig 2A) and audiometric damage was lacking (Fig 2B).

When analyzing outer hair cells specifically, quantified in 57 ears (Fig 3A), the degree of cell loss was typically similar to that of inner hair cells in any given ear (within 10\% of loss in the same ear in 38 of 56 ears). The degree of outer hair cell loss in one ear was within $10 \%$ of loss in the other ear in 10 out of 15 patients. This phenotype is exemplified by patient 29 , who suffered from autosomal dominant hereditary sensorineural hearing loss and progressive cataracts (Nadol et al., 1982). Specific loss of outer hair cells without a concomitant loss of inner hair cells was seen in 6 out of 56 ears, e.g. after acoustic trauma, as exemplified by patient 20 , who was missing $40 \%$ of outer hair cells (Nadol et al., 1982). As demonstrated for inner hair cells, an apparent correlation between histological (Fig 3A) and audiometric damage was lacking for outer hair cells (Fig 3B).

Strial atrophy, quantified in 100 cytocochleograms (Fig 4), ranged from none (in 20 
ears) to a maximal damage of $90 \%$ (in 1 ear, with 7 ears demonstrating over $80 \%$ damage). This highest percentage was observed in patient 73, who had been diagnosed with a viral labyrinthitis. Although patient 2 was diagnosed with the same condition bilaterally (otosclerosis), the stria in his left ear showed only $21 \%$ atrophy, while $50 \%$ of the stria on the right side was affected. In 23 out of 35 patients, strial atrophy in one ear was within $10 \%$ of strial atrophy in the other ear. Strial atrophy did not demonstrate an obvious correlation with histopathological damage (Fig 4A) or audiometric thresholds (Fig 4B).

Damage to spiral ganglion neurons was quantified in cytocochleograms from 127 ears, comprising the largest category in our collection (see Fig 5). As seen with other cell types, the degree of spiral ganglion neuron damage ranged from none (in 20 ears) to near total loss (in 1 ear, with 8 ears over $80 \%$ ). This maximal damage is represented by patient 83 , diagnosed with a meningioma of the cerebellopontine angle and subsequent degeneration of the cochlear nerve. About half of all patients showed a similar degree of neuronal damage in the two ears (23 of 44 patients within $10 \%$ difference). The largest discrepancy between two similarly diagnosed ears in a single individual was seen in patient 11, who was affected by Paget's disease and suffered $35 \%$ and $67 \%$ spiral ganglion neuron loss in the left and right ear, respectively. On visual inspection of cytocochleograms demonstrating spiral ganglion neuron loss (Fig 5A) and audiograms (Fig 5B), there was no robust correlation.

\subsection{Correlation between audiometric thresholds and cell}

\section{type-specific damage}

To quantify visual impressions from Figs 1-5, we calculated the Spearman's 
correlation coefficient between cell type-specific damage, as extracted from cytocochleograms, and audiometric thresholds measured at various frequencies (Fig $6)$.

Fig 6. Scatter plots of cell-specific damage versus audiometric thresholds.

Scatter plots of cell-specific damage inferred from cytocochleograms versus frequency.

Overall, the Spearman's correlation coefficient was at most 0.7 , and often below 0.5 , with 1.0 indicating perfect correlation. The correlation was most robust for inner hair cells at $250 \mathrm{~Hz}(0.67), 1000 \mathrm{~Hz}(0.67)$ and $2000 \mathrm{~Hz}(0.70)$, and poor for spiral ganglion neurons and stria vascularis across frequencies (see Table 1). The advanced age of our patient population explains some of the hearing loss in highfrequency regions (presbycusis), but this did not seem to have a major effect on the calculated values compared to other tonotopic areas. However, for several cell types, the correlation in the $8000 \mathrm{~Hz}$ region seemed to be slightly lower than in the rest of the cochlea.

\begin{tabular}{|c|c|c|c|c|c|c|}
\hline & & \multicolumn{5}{|c|}{ Cell type } \\
\hline \multirow{7}{*}{ 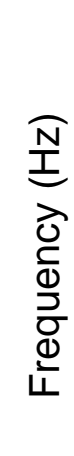 } & & $\mathrm{HC}$ & $\mathrm{IHC}$ & $\mathrm{OHC}$ & SGN & Stria \\
\hline & 250 & $0.38^{*}$ & $0.67^{*}$ & $0.53^{*}$ & 0.08 & $0.22^{*}$ \\
\hline & 500 & $0.42^{*}$ & $0.52^{*}$ & $0.48^{*}$ & 0.03 & $0.21^{*}$ \\
\hline & 1000 & $0.47^{*}$ & $0.67^{*}$ & $0.63^{*}$ & 0.04 & $0.30^{*}$ \\
\hline & 2000 & $0.45^{*}$ & $0.70^{*}$ & $0.49^{*}$ & 0.02 & $0.26^{*}$ \\
\hline & 4000 & $0.49^{*}$ & $0.52^{*}$ & $0.40^{*}$ & 0.08 & $0.24^{*}$ \\
\hline & 8000 & $0.41^{*}$ & $0.49^{*}$ & $0.32^{*}$ & -0.06 & 0.11 \\
\hline \multicolumn{2}{|c|}{ Word recognition } & $0.37^{*}$ & $0.38^{*}$ & 0.23 & 0.10 & 0.27 \\
\hline
\end{tabular}




\section{Table 1. Spearman's correlation coefficient between audiometric thresholds}

and degree of cell-specific damage. All values adjusted based on the BenjaminiHochberg correction for multiple comparisons. $\mathrm{HC}=$ hair cells, $\mathrm{IHC}=$ inner hair cells, $\mathrm{OHC}=$ outer hair cells, $\mathrm{SGN}=$ spiral ganglion neurons. ${ }^{*}$ indicates $\mathrm{p}<0.05$.

When correlating word recognition scores and cell type-specific damage in weighted frequency regions that make the most significant contributions to speech discrimination (Fig 7), Spearman's correlation coefficient was at most 0.38 . Sex- and age-specific analyses for all data revealed similar results. Additionally, alternative methods of assessing damage contributing to word recognition (e.g. by including the whole length of the cochlea or the area from $250 \mathrm{~Hz}$ to $4000 \mathrm{~Hz}$ ) failed to reveal a significant correlation.

\section{Fig 7. Scatter plots of cell-specific damage versus audiometric thresholds.}

Scatter plots of cell-specific damage inferred from cytocochleograms versus word recognition score.

To underscore the fact that large discrepancies at the cellular level may not be reflected in audiometric thresholds, we highlight patient 28 , a 53-year-old male with neurofibromatosis type 1 who was also diagnosed with bilateral presbycusis and right-sided Paget's disease. His last hearing test (4 years prior to death) revealed a similar bilateral high-frequency hearing loss with minor differences between the two ears (at most a $20 \mathrm{~dB}$ difference across all six tested frequencies). However, while the right ear showed no damage in any of the analyzed structures, the left ear demonstrated substantial damage of neurons (33\% loss) and the stria vascularis (15\% loss).

\section{Discussion}


Clinicians have long been aware that hearing performance as measured by an audiogram differs significantly from the hearing experience of a patient in everyday life. When fitting hearing aids, the implications of "dead" regions have been recognized for decades (Halpin et al., 1994; Moore, 2001). For example, patients with auditory neuropathy encounter severe difficulties when listening to speech in noise, though their audiometric thresholds in quiet are typically normal or near normal (Moser et al., 2013). Additionally, experiments in chinchillas have demonstrated that an extensive loss of inner hair cells (exceeding $80 \%$ ) produces only small effects on audiometric thresholds (Lobarinas et al., 2013). Nevertheless, an overwhelming proportion of human studies focus on using standardized hearing tests to verify the outcome of a certain intervention. Existing data derived from human temporal bones have suggested that the correlation between audiometric thresholds and cellular damage is low, but most of these studies focus only on presbycusis, and the number of samples is relatively small (Engstrom et al., 1987; Jennings et al., 2001; Kusunoki et al., 2004; Nelson et al., 2003; Suga et al., 1976; Suzuki et al., 2006).

\section{Conclusions}

Since our data suggest that neither frequency-specific thresholds nor word recognition scores as measured by the audiogram show a large enough correlation with histologically quantified cochlear damage to produce a viable assessment of patient hearing, developing novel diagnostic approaches is essential. Although the cochlea's small size and bony capsule make intracochlear interrogation challenging, better non- or minimally-invasive tools to diagnose cellular damage in the inner ear are needed. Promising new technologies soon expected to enter clinical applications include confocal fluorescence microscopy, optical coherence tomography, and in 
particular two-photon micro-endoscopy, which has been shown in animals to enable the detection of cell-specific damage in situ (Chen et al., 2007; MacDonald et al., 2008; Wong et al., 2000; Yang et al., 2013). Our results suggest that exclusive reliance on audiometric thresholds or word recognition scores in clinical trials of novel therapies for deafness, such as gene therapy, may prematurely disqualify a promising therapy when the current methods for detecting a potentially significant effect are not adequately sensitive. Our results strongly motivate the development of more sensitive and cell-specific diagnostic tools to complement and augment the current diagnostic armamentarium, and to precisely monitor cell type-specific response to treatment.

\section{Acknowledgments}

This work was supported by the Bertarelli Foundation (to K.M.S. and D.P.), Wyss Center Geneva (to K.M.S. and D.P.), Department of Defense Grant W81XWH-15-10472 (K.M.S.), Nancy Sayles Day Foundation (to K.M.S.) and Lauer Tinnitus Research Center (K.M.S.). The funding sources had no impact on the collection, analysis and interpretation of data, in the writing of the report and in the decision to submit the article for publication. The authors declare no competing interests. We would like to acknowledge the help of Haobing Wang, M.A. and Andrew C. Lysaght, Ph.D. with the creation of the graphs, and Garyfallia Pagonis, Mohammed Seyyedi, M.D. and Joseph B. Nadol, Jr., M.D. for their support with the identification of patients in the Massachusetts Eye and Ear Infirmary temporal bone collection. We thank Christopher F. Halpin, Ph.D. and Jessica E. Sagers, B.A. for valuable comments regarding the manuscript as well as Harvard Catalyst for statistical consultation. 
Harvard Catalyst | The Harvard Clinical and Translational Science Center (National Center for Research Resources and the National Center for Advancing Translational Sciences, National Institutes of Health Award UL1 TR001102) is supported by financial contributions from Harvard University and its affiliated academic healthcare centers. The content is solely the responsibility of the authors and does not necessarily represent the official views of Harvard Catalyst, Harvard University and its affiliated academic healthcare centers, or the National Institutes of Health.

\section{References}

World Health Organization (WHO). Make Listening Safe. http://www.who.int/pbd/deafness/activities/MLS_main_infographic_A4_lowres for_web.pdf?ua=1. Accessed on August 12, 2015. [Online].

ClinicalTrials.gov. Safety, Tolerability and Efficacy for CGF166 in Patients With Bilateral Severe-to-profound Hearing Loss. http://clinicaltrials.gov/ct2/show/NCT02132130. Accessed on August 12, 2015.

ANSI. 1997. ANSI S3.5-1997. American National Standard Methods for the Calculation of the Speech Intelligibility Index. ANSI, New York.

Carlson, M.L., Driscoll, C.L., Gifford, R.H., McMenomey, S.O. 2012. Cochlear implantation: current and future device options. Otolaryngologic clinics of North America 45, 221-48.

Causey, G.D., Hood, L.J., Hermanson, C.L., Bowling, L.S. 1984. The Maryland CNC test: normative studies. Audiology 23, 552-68.

Chen, F., Choudhury, N., Zheng, J., Matthews, S., Nutall, A.L., Jacques, S.L. 2007. In vivo imaging and low-coherence interferometry of organ of Corti vibration. Journal of biomedical optics 12, 021006.

Chien, W.W., Monzack, E.L., McDougald, D.S., Cunningham, L.L. 2015. Gene therapy for sensorineural hearing loss. Ear and hearing 36, 1-7.

Daniel, E. 2007. Noise and hearing loss: a review. The Journal of school health 77, 22531.

Engstrom, B., Hillerdal, M., Laurell, G., Bagger-Sjoback, D. 1987. Selected pathological findings in the human cochlea. Acta oto-laryngologica. Supplementum 436, 1106.

Fletcher, H. 1953. Speech and Hearing in Communication Van Nostrand, New York, NY.

Geleoc, G.S., Holt, J.R. 2014. Sound strategies for hearing restoration. Science 344, 1241062.

Gelfand, S.A. 1997. Essentials of Audiology Thieme Medical Publishers, Inc., New York, NY. 
Glasberg, B.R., Moore, B.C. 1990. Derivation of auditory filter shapes from notched-noise data. Hearing research 47, 103-38.

Greenwood, D.D. 1961a. Auditory Masking and the Critical Band. J Acoust Soc Am. 33, 484-502.

Greenwood, D.D. 1961b. Critical Bandwidth and the Frequency Coordinates of the Basilar Membrane. J Acoust Soc Am. 33, 1344-1356.

Halpin, C., Thornton, A., Hasso, M. 1994. Low-frequency sensorineural loss: clinical evaluation and implications for hearing aid fitting. Ear and hearing 15, 71-81.

Jennings, C.R., Jones, N.S. 2001. Presbyacusis. The Journal of laryngology and otology 115, 171-8.

Kusunoki, T., Cureoglu, S., Schachern, P.A., Baba, K., Kariya, S., Paparella, M.M. 2004. Agerelated histopathologic changes in the human cochlea: a temporal bone study. Otolaryngology--head and neck surgery : official journal of American Academy of Otolaryngology-Head and Neck Surgery 131, 897-903.

Lobarinas, E., Salvi, R., Ding, D. 2013. Insensitivity of the audiogram to carboplatin induced inner hair cell loss in chinchillas. Hearing research 302, 113-20.

MacDonald, G.H., Rubel, E.W. 2008. Three-dimensional imaging of the intact mouse cochlea by fluorescent laser scanning confocal microscopy. Hearing research 243, 1-10.

Moore, B.C. 1986. Parallels between frequency selectivity measured psychophysically and in cochlear mechanics. Scand Audiol Suppl. 25, 139-52.

Moore, B.C. 2001. Dead regions in the cochlea: diagnosis, perceptual consequences, and implications for the fitting of hearing AIDS. Trends in amplification 5, 1-34.

Moore, B.C., Glasberg, B.R. 1983. Suggested formulae for calculating auditory-filter bandwidths and excitation patterns. The Journal of the Acoustical Society of America 74, 750-3.

Moser, T., Predoehl, F., Starr, A. 2013. Review of hair cell synapse defects in sensorineural hearing impairment. Otology \& neurotology : official publication of the American Otological Society, American Neurotology Society [and] European Academy of Otology and Neurotology 34, 995-1004.

Nadol, J.B., Jr., Burgess, B. 1982. Cochleosaccular degeneration of the inner ear and progressive cataracts inherited as an autosomal dominant trait. The Laryngoscope 92, 1028-37.

Nelson, E.G., Hinojosa, R. 2003. Presbycusis: a human temporal bone study of individuals with flat audiometric patterns of hearing loss using a new method to quantify stria vascularis volume. The Laryngoscope 113, 1672-86.

Schuknecht, H. 1968. Temporal bone removal at autopsy. Preparation and uses. Archives of otolaryngology $87,129-37$.

Schuknecht, H. 1993. Pathology of the Ear, 2nd ed. Lea and Febiger, Philadelphia, PA. pp. 7-30.

Suga, F., Lindsay, J.R. 1976. Histopathological observations of presbycusis. The Annals of otology, rhinology, and laryngology $85,169-84$.

Suzuki, T., Nomoto, Y., Nakagawa, T., Kuwahata, N., Ogawa, H., Suzuki, Y., Ito, J., Omori, K. 2006. Age-dependent degeneration of the stria vascularis in human cochleae. The Laryngoscope 116, 1846-50.

Thornton, A.R., Raffin, M.J. 1978. Speech-discrimination scores modeled as a binomial variable. Journal of speech and hearing research 21, 507-18.

Wallhagen, M.I., Strawbridge, W.J., Cohen, R.D., Kaplan, G.A. 1997. An increasing prevalence of hearing impairment and associated risk factors over three decades of the Alameda County Study. American journal of public health 87, 440-2. 
Wong, B.J., de Boer, J.F., Park, B.H., Chen, Z., Nelson, J.S. 2000. Optical coherence tomography of the rat cochlea. Journal of biomedical optics 5, 367-70.

Yang, X., Pu, Y., Hsieh, C.L., Ong, C.A., Psaltis, D., Stankovic, K.M. 2013. Two-photon microscopy of the mouse cochlea in situ for cellular diagnosis. Journal of biomedical optics 18, 31104. 


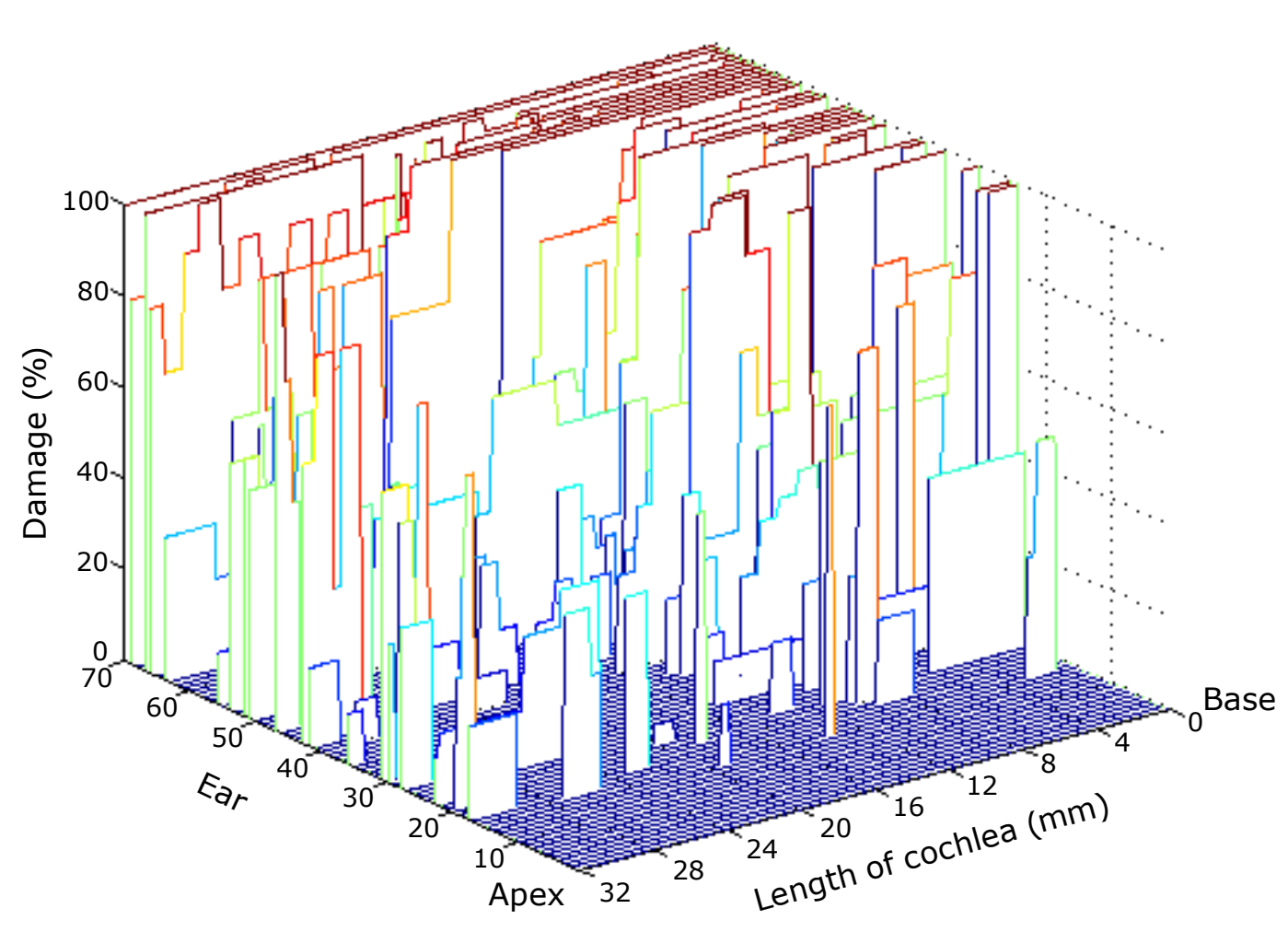

B

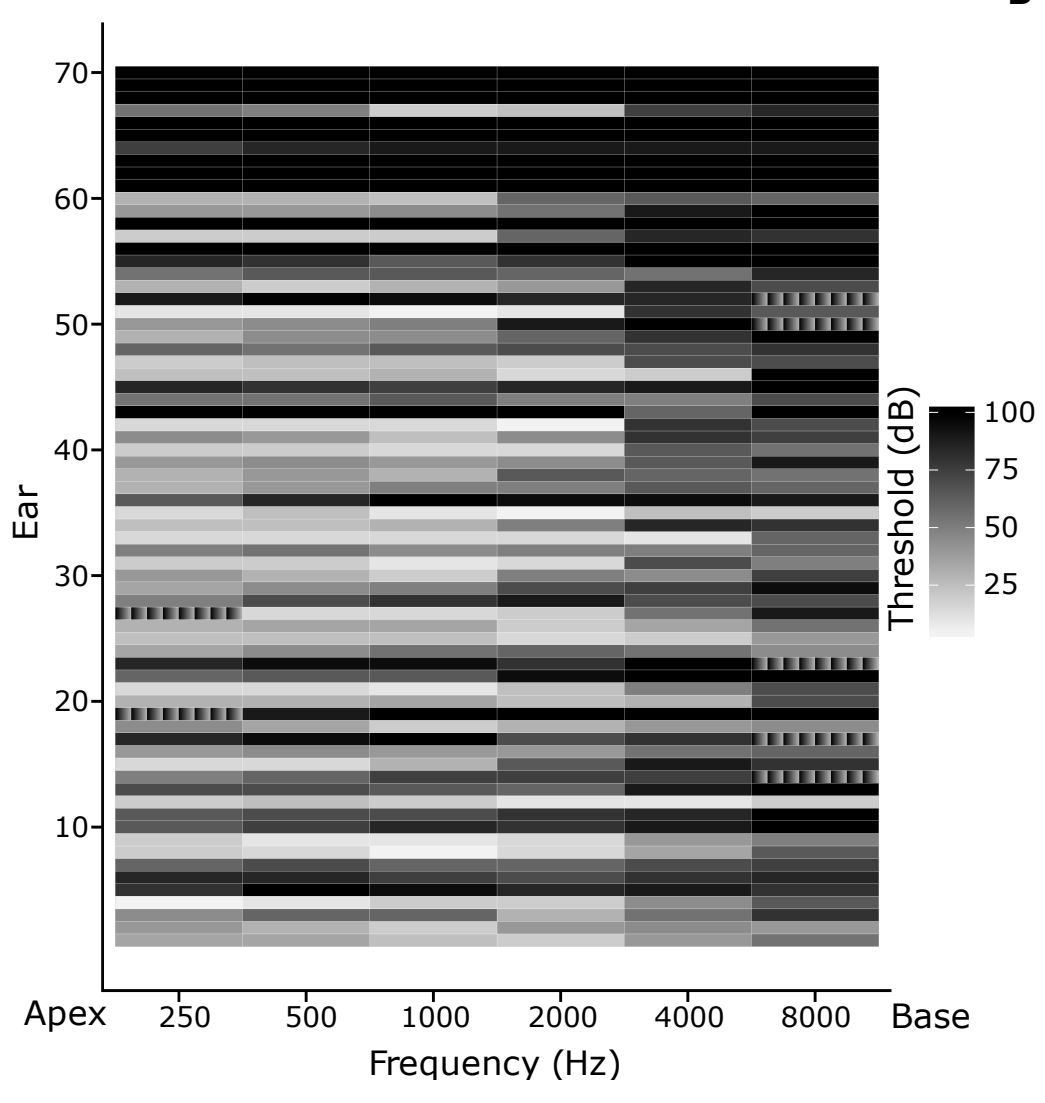




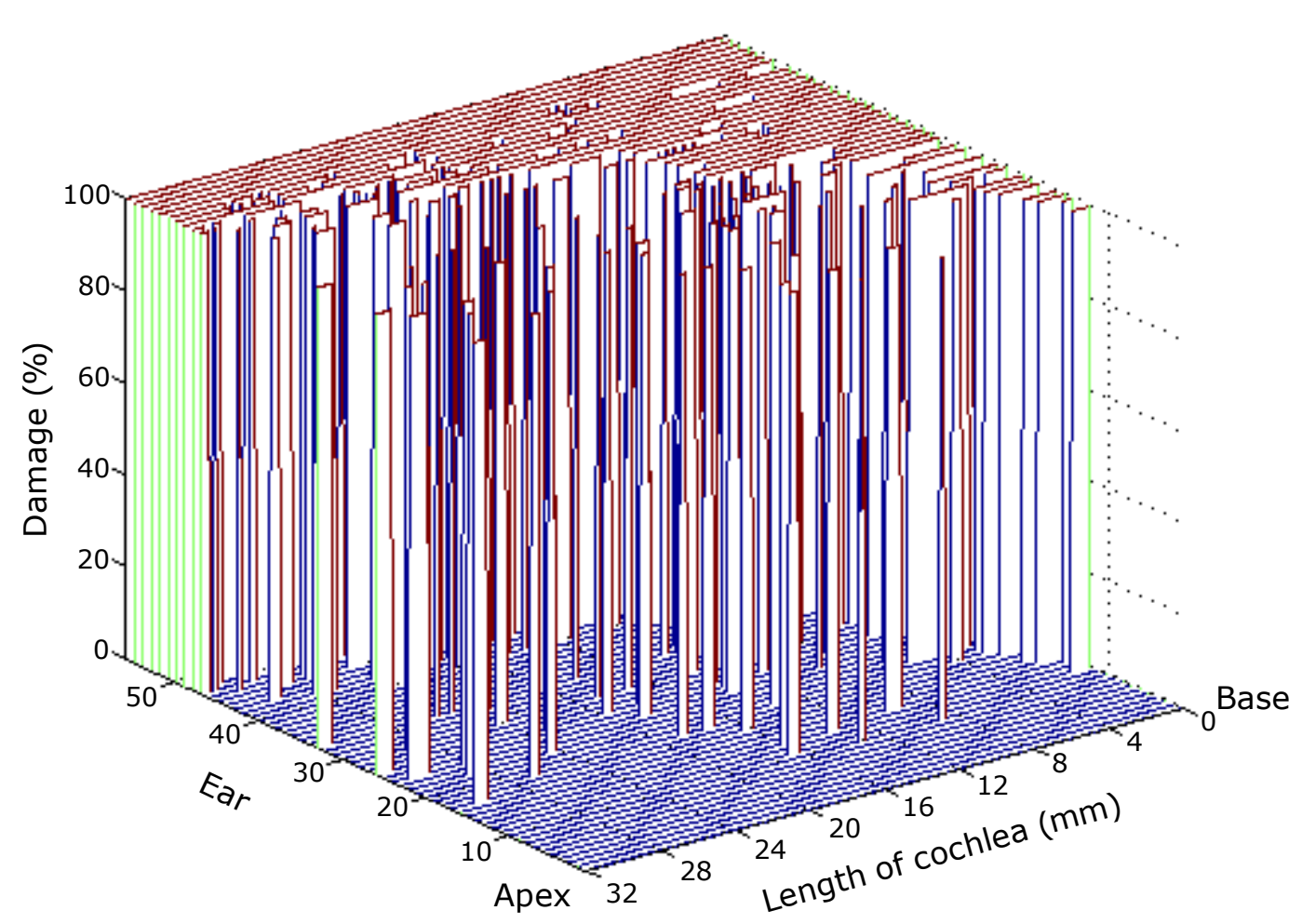

IHC

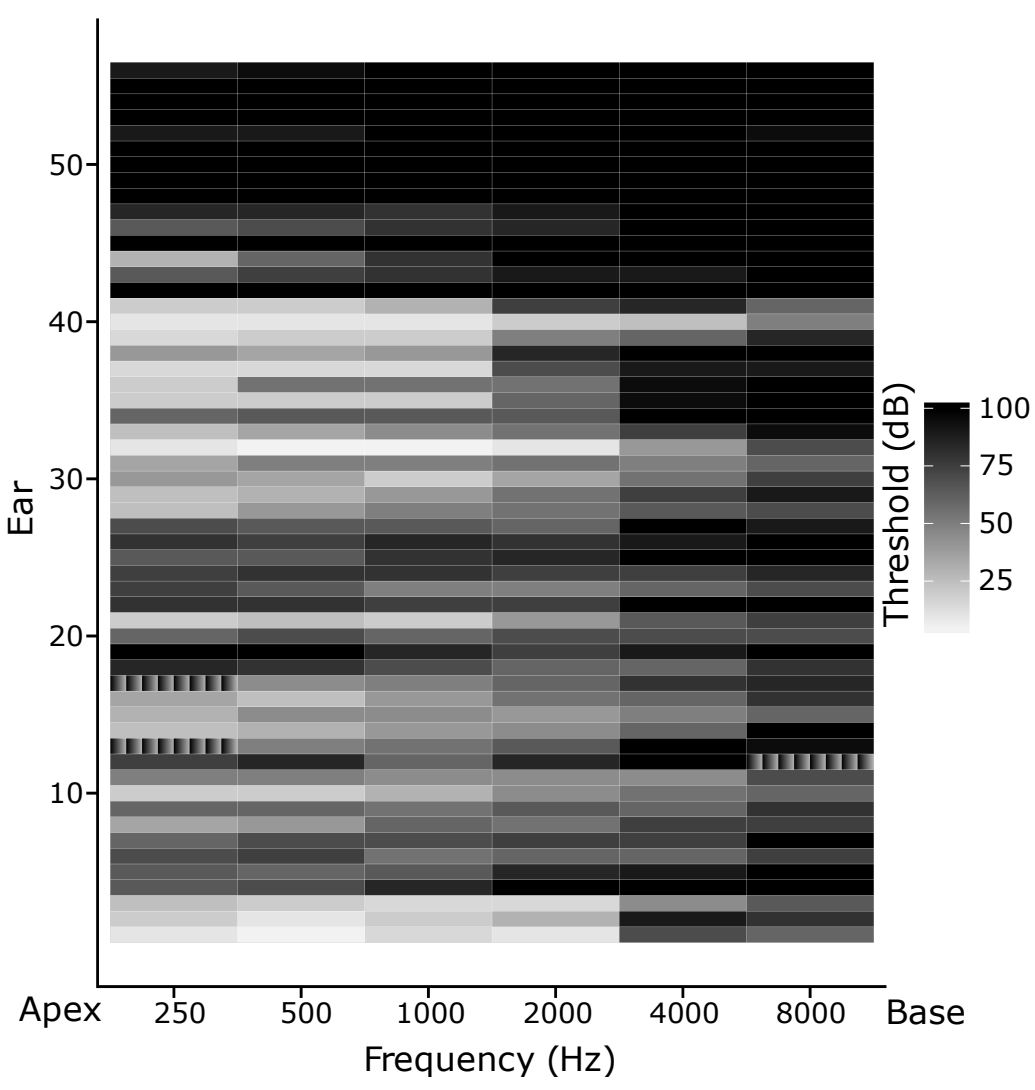


A

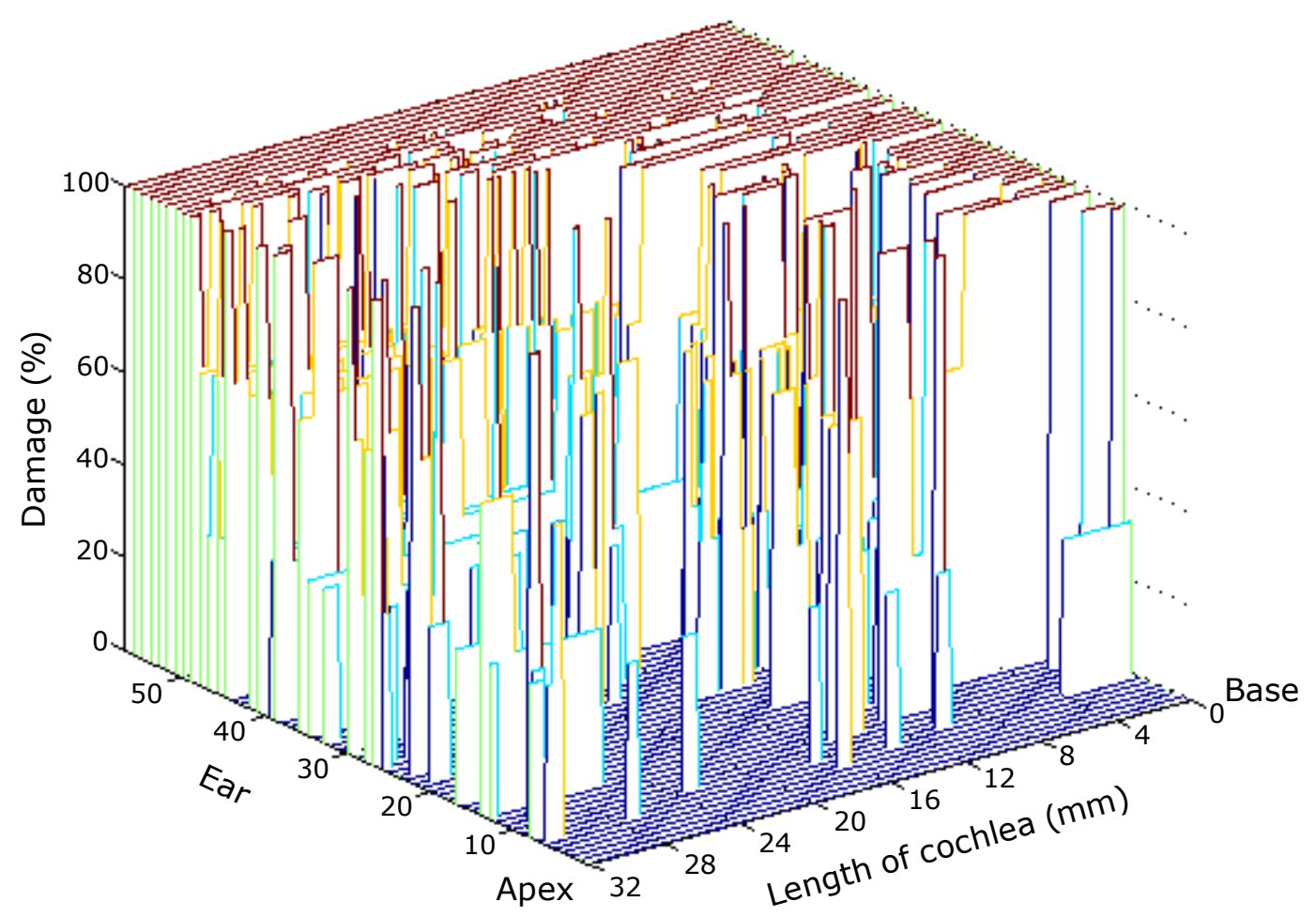

$\mathrm{OHC}$

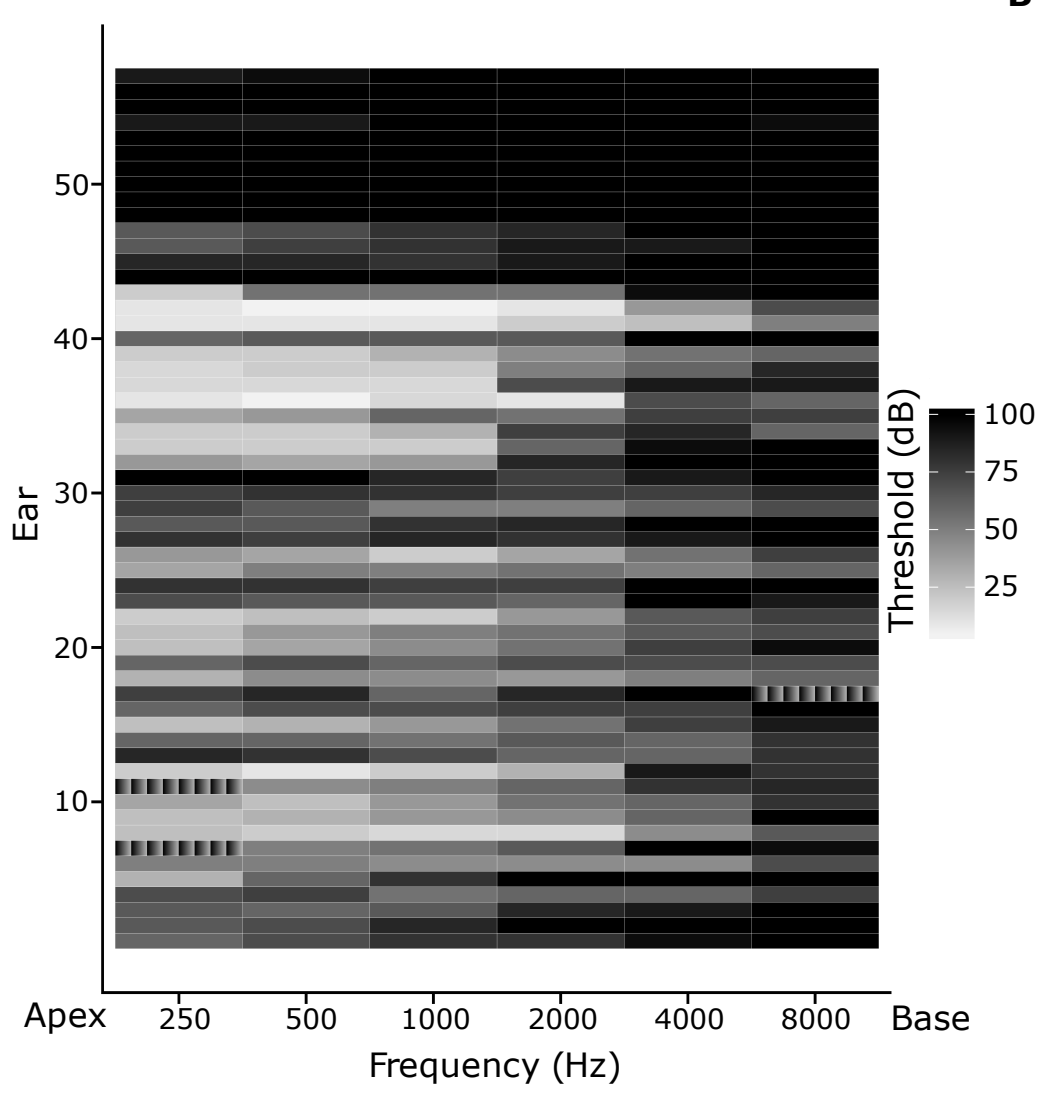



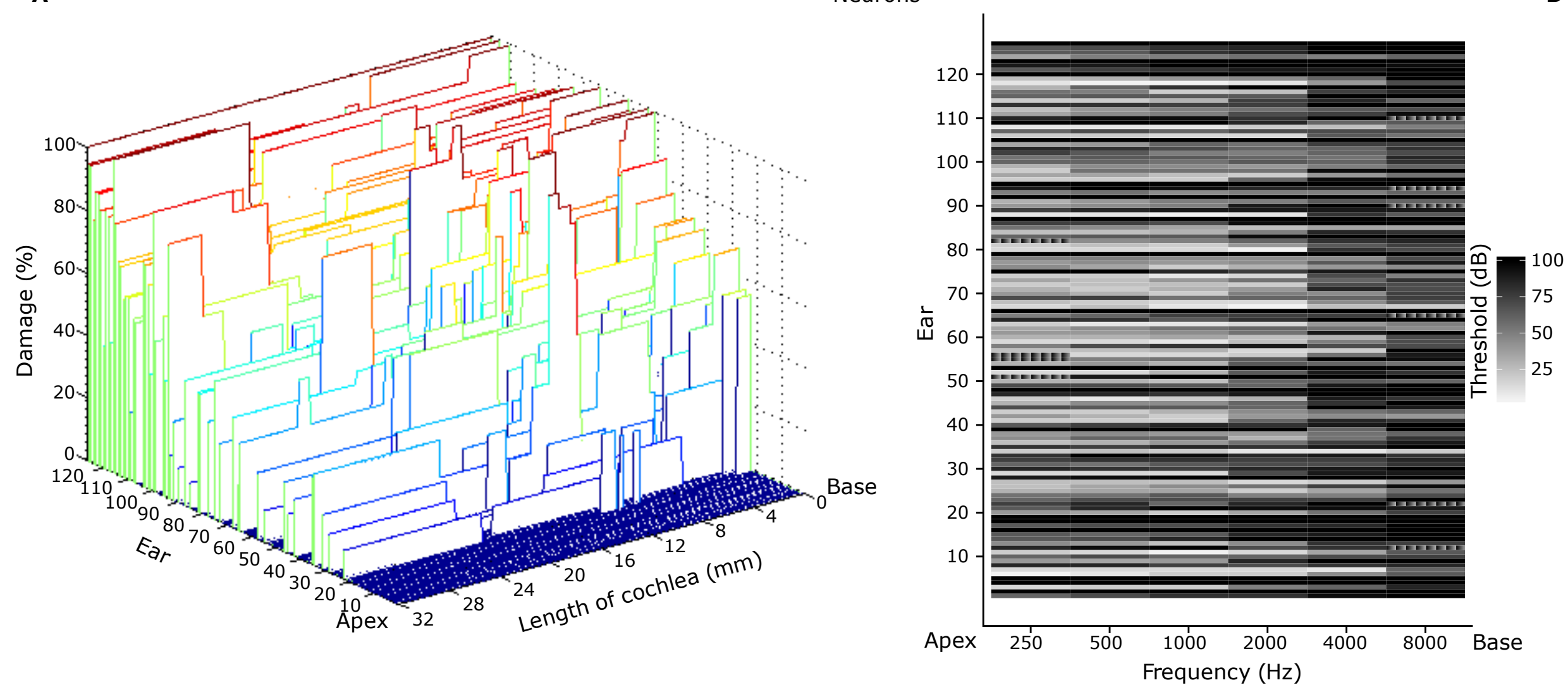


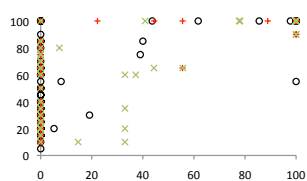

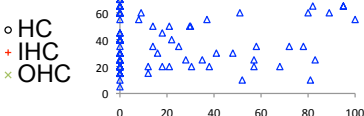
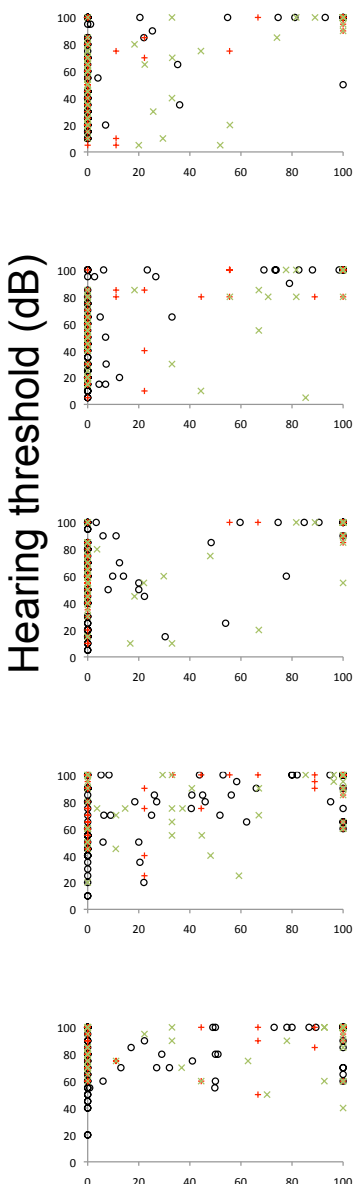

$\mathrm{HC}$ damage (\%)
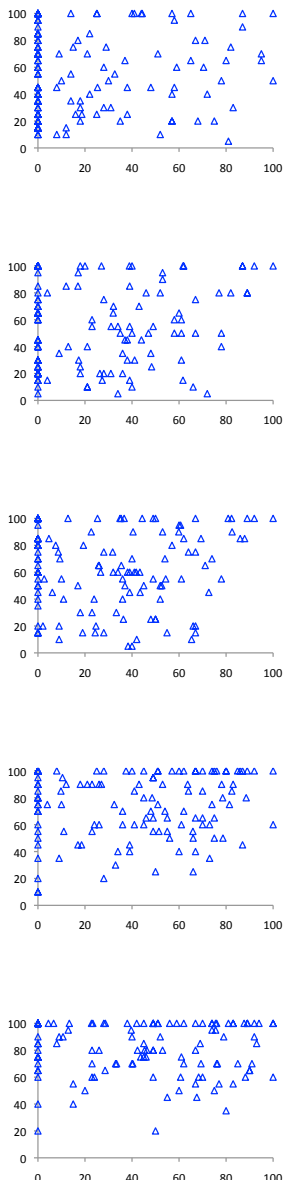
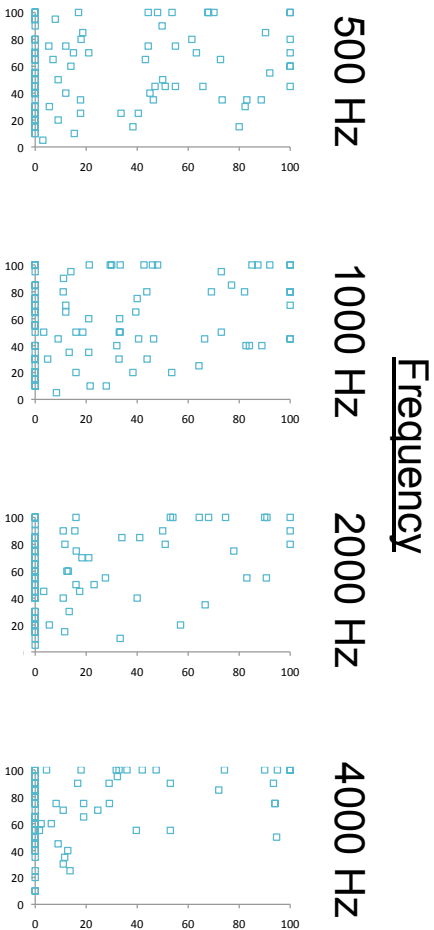

잉
존

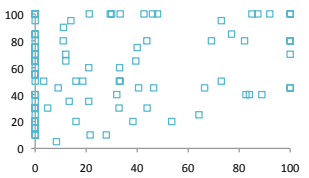

N
I
N

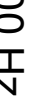



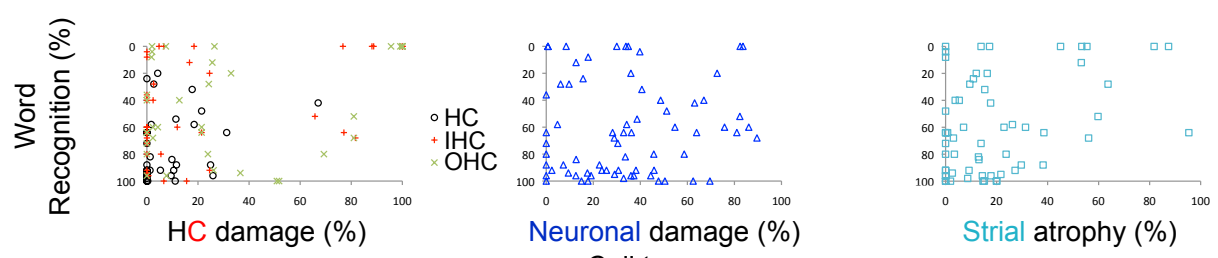

Cell type 
A. Generation of a waterfall plot from cytocochleograms

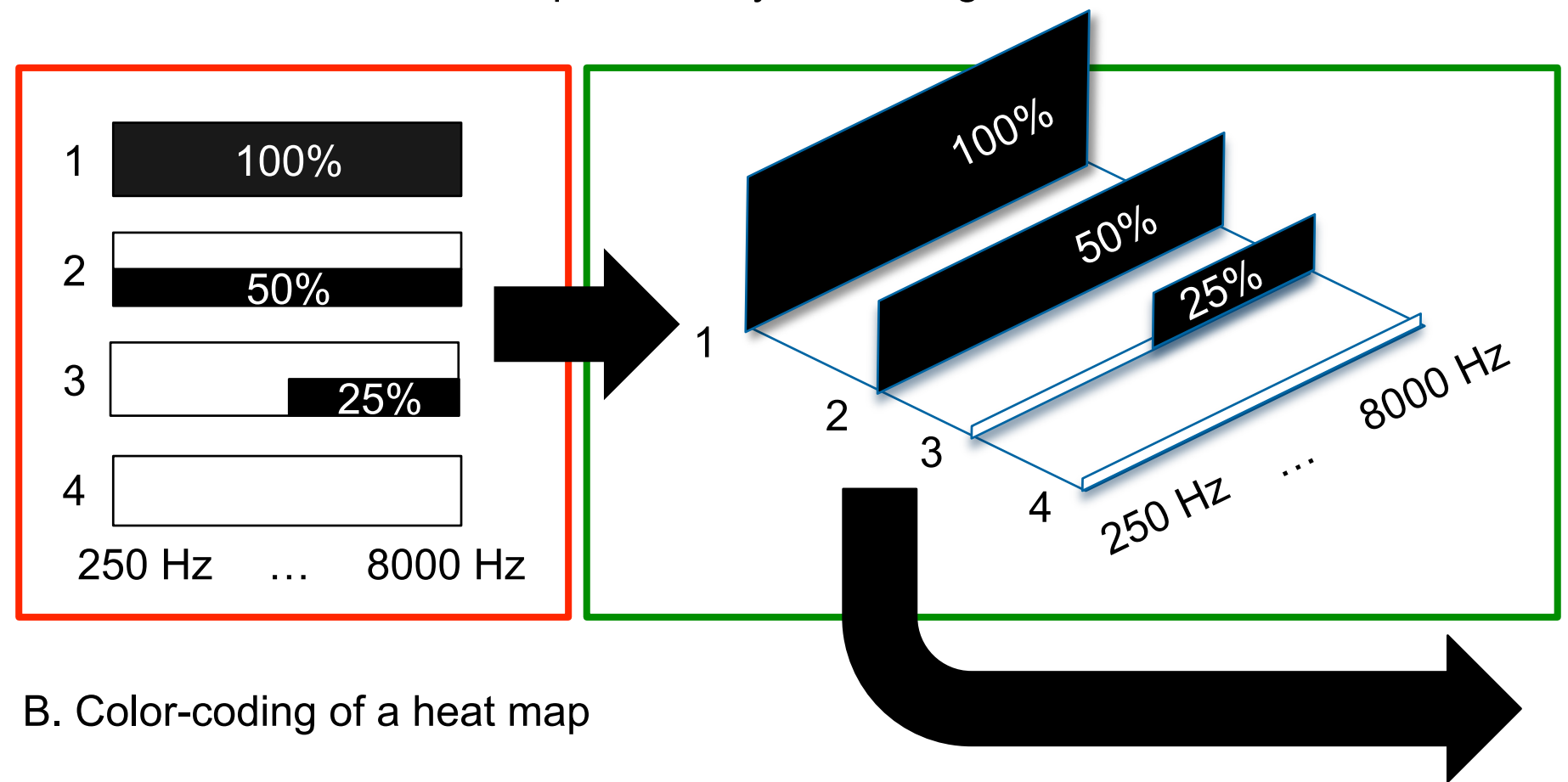

\begin{tabular}{|c|c|c|c|c|c|}
\hline $250 \mathrm{~Hz}$ & $500 \mathrm{~Hz}$ & $1000 \mathrm{~Hz}$ & $2000 \mathrm{~Hz}$ & $4000 \mathrm{~Hz}$ & $8000 \mathrm{~Hz}$ \\
\hline $0 \mathrm{~dB}$ & $20 \mathrm{~dB}$ & $40 \mathrm{~dB}$ & $60 \mathrm{~dB}$ & $80 \mathrm{~dB}$ & $100 \mathrm{~dB}$ \\
\hline$\downarrow$ & $\downarrow$ & & & & \\
\hline & & & & & \\
\hline
\end{tabular}

C. $100 \%$ correlation for example in "A":

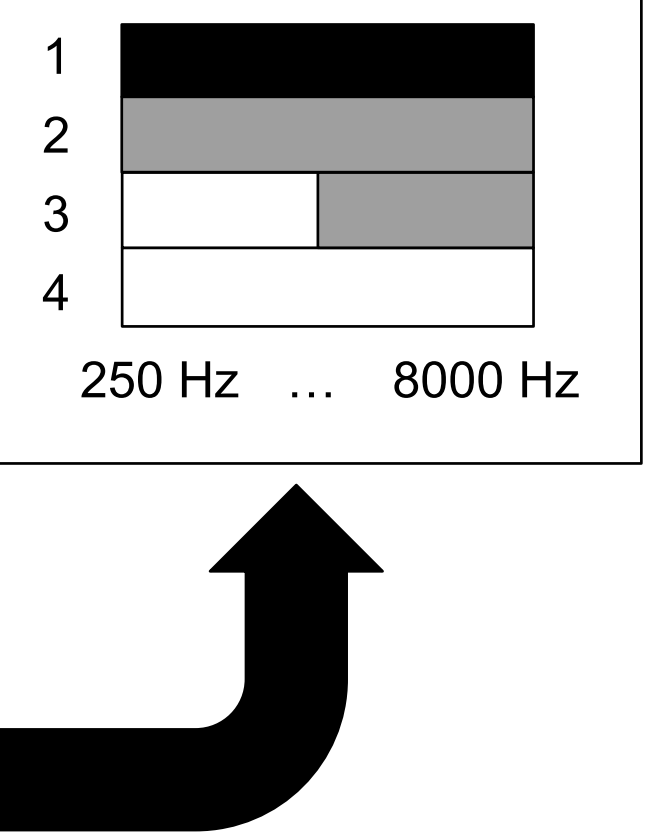


